\title{
Quantum-Chemical Modeling of the Non-Linear Graphenes from 7, 9, 10 Hexagons in the Framework of the Molecular ModelMethod of Mndo
}

\author{
Babkin $\mathrm{VA}^{1 *}$, Andreev $\mathrm{DS}^{2}$, Ignatov $\mathrm{AV}^{1}$, Bakholdin $\mathrm{II}^{1}$, Titova $\mathrm{ES}^{3}$, Rakhimov $\mathrm{AI}^{3}$, Rakhimova $\mathrm{NA}^{3}$, Fedunov RG ${ }^{4}$, \\ Lebedev NG4 and Zaikov GE ${ }^{1}$
}

${ }^{1}$ Department of Chemical Sciences, Volgograd State Technical University, Russia

${ }^{2}$ Department Mathematical and Natural Sciences, Volgograd State Technical University, Russia

${ }^{3}$ Department of Organic Chemistry, Volgograd State of Technical University, Russia

${ }^{4}$ Department of Theoretical physics and wave processes, Volgograd State of Technical University, Russia

Submission: July 01, 2017; Published: September 25, 2017

*Corresponding author: Babkin VA, Department of Chemical Sciences, Volgograd State Technical University, Russia, Email: Babkin_v.a@mail.ru

\begin{abstract}
The quantum-chemical calculation of the non-linear graphene molecules from 7, 9 and 10 hexagons by the MNDO method with optimization of geometry for all parameters by the standard gradient method within the framework of the molecular nonlinear graphene model was performed for the first time. The optimized geometric and electronic structure of these compounds is obtained. The acid strength (pKa $=33$ ) of the studied graphenes is theoretically estimated. The molecules of 7, 9 and 10 hexagons belong to the class of very weak acids (pKa> 14). It is shown that an increase in the number of hexagons reduces the Harte energy of the studied graphenes.
\end{abstract}

Keywords:Quantum-chemical calculation; MNDO method; Grapheme molecules of 7, 9 and 10 hexagons; Acid strength

\section{Introduction}

Quantum-chemical calculations of the nonlinear graphenes from 5-6 hexagons within the framework of the molecular model by the MNDO method for the first time were performed with optimization of geometry for all parameters in [1]. In order to obtain new regularities and, in particular, the energies of the highest occupied molecular orbital energy $\left(\mathrm{E}_{\text {номо }}\right)$, the energy of the lowest unoccupied molecular orbital energy $\left(\mathrm{E}_{\mathrm{Luм0}}\right)$ of the studied graphenes, depending on the hexagons under research, it is reasonable to continue these calculations for 7-10 and so on hexagons, which will allow us to estimate their electronic conductivity. In connection with this, the goal of the present paper is the quantum-chemical calculation of the nonlinear graphenes from 7-10 hexagons within the framework of the molecular model.

\section{Methodical Part}

The MNDO method, which reproduces the energy characteristics of molecules fairly well [2], was chosen for the quantum-chemical calculation of the non-linear graphenes from 7, 9 and 10 hexagons (it is impossible to build a model from 8 hexagons that is adequate to graphene) within the framework of the molecular graphene model.

Optimization of the geometry of the models was carried out in all parameters by the standard gradient method built into Firefly [3], which is partly based on the GAMESS source code [4], in the approximation of the isolated molecules in the gas phase. The acid strength of the studied molecules was estimated using the formula $\mathrm{pKa}=42.11-147.18 \mathrm{qmax}^{\mathrm{H}+}[5]$ (where $\mathrm{qmax}^{\mathrm{H+}}$ is the maximum charge on the hydrogen atom, pKa is the universal acidity index). The program MacMolPlt [6] was used to visualize the model of the molecules. These graphenes are shown schematically in Figure1.

\section{Results of Calculations}

The optimized geometric and electronic structure, total energy (E0) and electron energy $\left(\mathrm{E}_{\mathrm{EL}}\right)$, the highest occupied molecular orbital energy $\left(\mathrm{E}_{\text {номо }}\right)$, the lowest unoccupied molecular orbital energy $\left(\mathrm{E}_{\text {Lumo }}\right)$ and the Harte energy $(\mathrm{EH})$ 
as a criterion for the electronic conductivity of the molecules of the researched graphenes, obtained by the MNDO method, are shown in Figure 1-4 and Table 1-4. Using the formula pKa $=42.11-147.18 \mathrm{qmax}^{\mathrm{H}+}$ [5] $\left(\mathrm{qmax}^{\mathrm{H}+}=+0.06-\right.$ the maximum charge on the hydrogen atom, the same for all three models,
pKa is the universal acidity index, see Table 1-3, we find the value of the acid force pKa, Equal to 33. In addition, Table 4 shows, that the addition of three hexogons to the polycyclic hydrocarbon reduces the Hartree energy from $681 \mathrm{~kJ} / \mathrm{mol}$ to $583 \mathrm{~kJ} / \mathrm{mol}$, or from $7.1 \mathrm{eV}$ to $6 \mathrm{eV}$ [7].

Table 1: Optimized bond lengths, valence angles and atom charges of the the non-linear graphene of 7 hexagons.

\begin{tabular}{|c|c|c|c|c|c|}
\hline Bond Lengths & $\mathrm{R}, \mathrm{A}$ & Valence Angles & Grad & Atom & Atomic Charge \\
\hline$C(2)-C(1)$ & 1.44 & $C(5)-C(4)-C(1)$ & 121 & $\mathrm{C}(1)$ & -0.04 \\
\hline $\mathrm{C}(3)-\mathrm{C}(2)$ & 1.42 & $C(6)-C(3)-C(2)$ & 120 & $\mathrm{C}(2)$ & -0.03 \\
\hline$C(3)-C(6)$ & 1.45 & $C(1)-C(2)-C(3)$ & 119 & $\mathrm{C}(3)$ & -0.01 \\
\hline$C(4)-C(1)$ & 1.38 & $C(5)-C(6)-C(3)$ & 120 & $\mathrm{C}(4)$ & -0.04 \\
\hline$C(4)-C(5)$ & 1.44 & $C(7)-C(2)-C(3)$ & 119 & $C(5)$ & -0.03 \\
\hline$C(5)-C(14)$ & 1.44 & $C(11)-C(6)-C(3)$ & 120 & $C(6)$ & -0.01 \\
\hline$C(6)-C(5)$ & 1.42 & $C(2)-C(1)-C(4)$ & 121 & $\mathrm{C}(7)$ & -0.04 \\
\hline$C(7)-C(2)$ & 1.44 & $C(14)-C(5)-C(4)$ & 123 & $\mathrm{C}(8)$ & -0.04 \\
\hline$C(8)-C(7)$ & 1.38 & $C(6)-C(5)-C(4)$ & 119 & $\mathrm{C}(9)$ & -0.01 \\
\hline$C(8)-C(10)$ & 1.44 & $C(13)-C(14)-C(5)$ & 121 & $\mathrm{C}(10)$ & -0.03 \\
\hline$C(9)-C(3)$ & 1.45 & $C(14)-C(5)-C(6)$ & 119 & $\mathrm{C}(11)$ & -0.01 \\
\hline$C(10)-C(9)$ & 1.42 & $C(1)-C(2)-C(7)$ & 123 & $\mathrm{C}(12)$ & -0.03 \\
\hline$C(10)-C(19)$ & 1.44 & $\mathrm{C}(10)-\mathrm{C}(8)-\mathrm{C}(7)$ & 121 & $\mathrm{C}(13)$ & -0.04 \\
\hline$C(11)-C(6)$ & 1.45 & $C(2)-C(7)-C(8)$ & 121 & $\mathrm{C}(14)$ & -0.04 \\
\hline $\mathrm{C}(12)-\mathrm{C}(11)$ & 1.42 & $C(9)-C(10)-C(8)$ & 119 & $\mathrm{C}(15)$ & -0.01 \\
\hline $\mathrm{C}(13)-\mathrm{C}(12)$ & 1.44 & $C(19)-C(10)-C(8)$ & 123 & $C(16)$ & -0.01 \\
\hline$C(14)-C(13)$ & 1.38 & $\mathrm{C}(2)-\mathrm{C}(3)-\mathrm{C}(9)$ & 120 & $\mathrm{C}(17)$ & -0.03 \\
\hline$C(15)-C(11)$ & 1.45 & $C(6)-C(3)-C(9)$ & 120 & $\mathrm{C}(18)$ & -0.04 \\
\hline$C(15)-C(16)$ & 1.45 & $C(19)-C(10)-C(9)$ & 119 & $\mathrm{C}(19)$ & -0.04 \\
\hline$C(16)-C(9)$ & 1.45 & $\mathrm{C}(3)-\mathrm{C}(9)-\mathrm{C}(10)$ & 120 & $\mathrm{C}(20)$ & -0.04 \\
\hline$C(17)-C(16)$ & 1.42 & $\mathrm{C}(18)-\mathrm{C}(19)-\mathrm{C}(10)$ & 121 & $\mathrm{C}(21)$ & -0.04 \\
\hline$C(17)-C(24)$ & 1.44 & $C(16)-C(9)-C(10)$ & 120 & $C(22)$ & -0.03 \\
\hline $\mathrm{C}(18)-\mathrm{C}(17)$ & 1.44 & $C(5)-C(6)-C(11)$ & 120 & $\mathrm{C}(23)$ & -0.04 \\
\hline $\mathrm{C}(19)-\mathrm{C}(18)$ & 1.38 & $C(16)-C(15)-C(11)$ & 120 & $\mathrm{C}(24)$ & -0.04 \\
\hline$C(20)-C(12)$ & 1.44 & $C(6)-C(11)-C(12)$ & 120 & $\mathrm{H}(25)$ & +0.06 \\
\hline$C(21)-C(20)$ & 1.38 & $\mathrm{C}(15)-\mathrm{C}(11)-\mathrm{C}(12)$ & 120 & $\mathrm{H}(26)$ & +0.06 \\
\hline$C(21)-C(22)$ & 1.44 & $\mathrm{C}(11)-\mathrm{C}(12)-\mathrm{C}(13)$ & 119 & $\mathrm{H}(27)$ & +0.06 \\
\hline$C(22)-C(15)$ & 1.42 & $\mathrm{C}(20)-\mathrm{C}(12)-\mathrm{C}(13)$ & 123 & $\mathrm{H}(28)$ & +0.06 \\
\hline$C(23)-C(22)$ & 1.44 & $C(12)-C(13)-C(14)$ & 121 & $\mathrm{H}(29)$ & +0.06 \\
\hline$C(24)-C(23)$ & 1.38 & $C(6)-C(11)-C(15)$ & 120 & $\mathrm{H}(30)$ & +0.06 \\
\hline $\mathrm{H}(25)-\mathrm{C}(4)$ & 1.09 & $\mathrm{C}(9)-\mathrm{C}(16)-\mathrm{C}(15)$ & 120 & $\mathrm{H}(31)$ & +0.06 \\
\hline $\mathrm{H}(26)-\mathrm{C}(1)$ & 1.09 & $\mathrm{C}(17)-\mathrm{C}(16)-\mathrm{C}(15)$ & 120 & $\mathrm{H}(32)$ & +0.06 \\
\hline $\mathrm{H}(27)-\mathrm{C}(7)$ & 1.09 & $C(3)-C(9)-C(16)$ & 120 & $\mathrm{H}(33)$ & +0.06 \\
\hline $\mathrm{H}(28)-\mathrm{C}(8)$ & 1.09 & $C(24)-C(17)-C(16)$ & 119 & $\mathrm{H}(34)$ & +0.06 \\
\hline $\mathrm{H}(29)-\mathrm{C}(19)$ & 1.09 & $C(9)-C(16)-C(17)$ & 120 & $\mathrm{H}(35)$ & +0.06 \\
\hline $\mathrm{H}(30)-\mathrm{C}(18)$ & 1.09 & $C(23)-C(24)-C(17)$ & 121 & $\mathrm{H}(36)$ & +0.06 \\
\hline $\mathrm{H}(31)-\mathrm{C}(14)$ & 1.09 & $\mathrm{C}(16)-\mathrm{C}(17)-\mathrm{C}(18)$ & 119 & & \\
\hline $\mathrm{H}(32)-\mathrm{C}(13)$ & 1.09 & $\mathrm{C}(24)-\mathrm{C}(17)-\mathrm{C}(18)$ & 122 & & \\
\hline $\mathrm{H}(33)-\mathrm{C}(20)$ & 1.09 & $C(17)-C(18)-C(19)$ & 121 & & \\
\hline
\end{tabular}


Recent Advances in Petrochemical Science

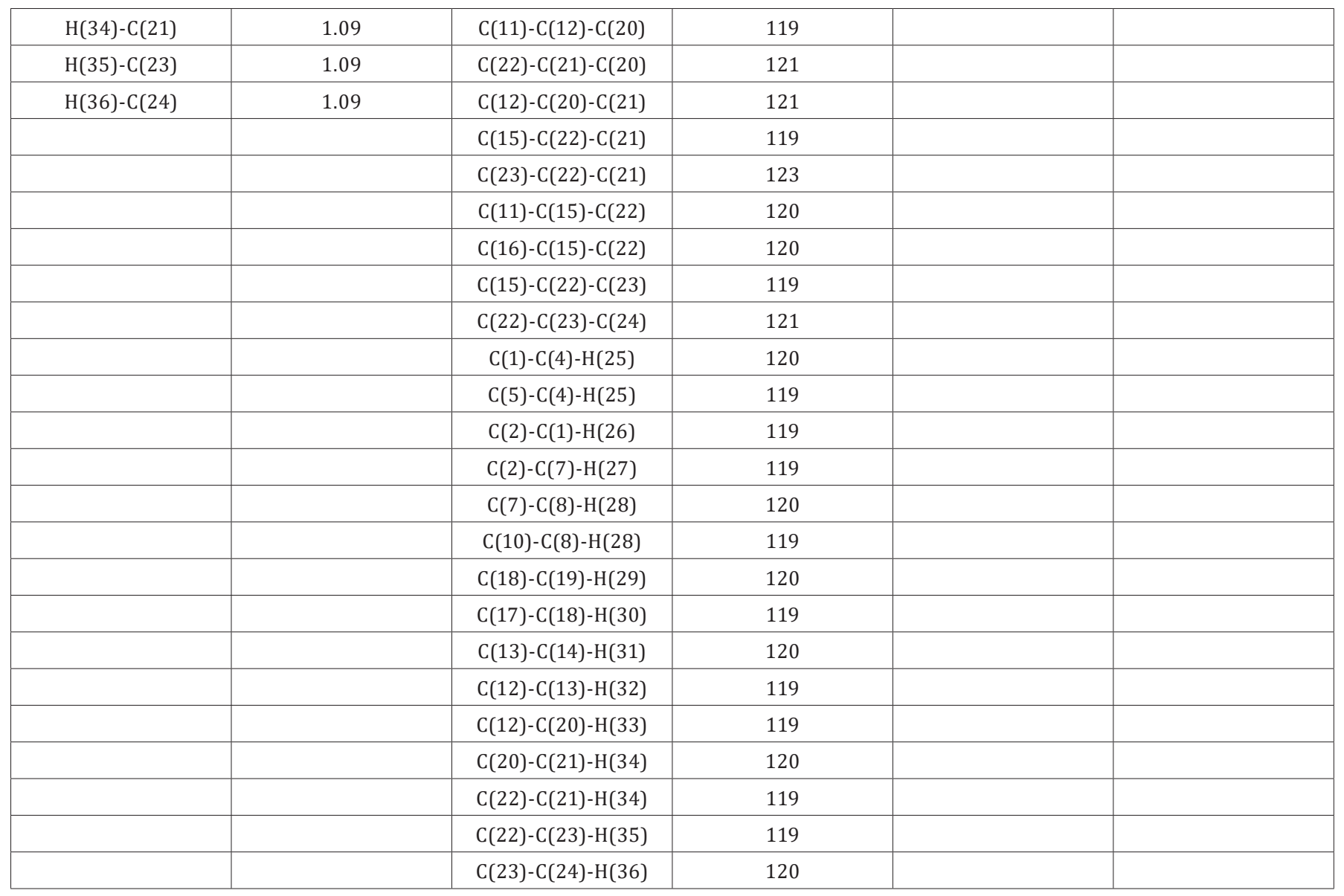

Table 2: Optimized bond lengths, valence angles and atom charges of the the non-linear graphene of 9 hexagons.

\begin{tabular}{|c|c|c|c|c|c|}
\hline Bond Lengths & $\mathbf{R}, \mathbf{A}$ & Valence Angles & Grad & Atom & Atomic Charge \\
\hline $\mathrm{C}(2)-\mathrm{C}(1)$ & 1.47 & $\mathrm{C}(5)-\mathrm{C}(4)-\mathrm{C}(1)$ & 120 & $\mathrm{C}(1)$ & -0.02 \\
\hline $\mathrm{C}(3)-\mathrm{C}(2)$ & 1.42 & $\mathrm{C}(39)-\mathrm{C}(37)-\mathrm{C}(1)$ & 122 & $\mathrm{C}(2)$ & -0.01 \\
\hline $\mathrm{C}(3)-\mathrm{C}(6)$ & 1.46 & $\mathrm{C}(6)-\mathrm{C}(3)-\mathrm{C}(2)$ & 121 & $\mathrm{C}(3)$ & -0.01 \\
\hline $\mathrm{C}(4)-\mathrm{C}(1)$ & 1.44 & $\mathrm{C}(1)-\mathrm{C}(2)-\mathrm{C}(3)$ & 120 & $\mathrm{C}(4)$ & -0.01 \\
\hline $\mathrm{C}(4)-\mathrm{C}(5)$ & 1.46 & $\mathrm{C}(5)-\mathrm{C}(6)-\mathrm{C}(3)$ & 120 & $\mathrm{C}(5)$ & 0.00 \\
\hline $\mathrm{C}(5)-\mathrm{C}(14)$ & 1.44 & $\mathrm{C}(7)-\mathrm{C}(2)-\mathrm{C}(3)$ & 118 & $\mathrm{C}(6)$ & -0.01 \\
\hline $\mathrm{C}(6)-\mathrm{C}(5)$ & 1.42 & $\mathrm{C}(11)-\mathrm{C}(6)-\mathrm{C}(3)$ & 120 & $\mathrm{C}(7)$ & -0.04 \\
\hline $\mathrm{C}(7)-\mathrm{C}(2)$ & 1.43 & $\mathrm{C}(16)-\mathrm{C}(9)-\mathrm{C}(3)$ & 121 & $\mathrm{C}(8)$ & -0.04 \\
\hline $\mathrm{C}(8)-\mathrm{C}(7)$ & 1.38 & $\mathrm{C}(2)-\mathrm{C}(1)-\mathrm{C}(4)$ & 119 & $\mathrm{C}(9)$ & -0.01 \\
\hline $\mathrm{C}(8)-\mathrm{C}(10)$ & 1.43 & $\mathrm{C}(14)-\mathrm{C}(5)-\mathrm{C}(4)$ & 120 & $\mathrm{C}(10)$ & -0.03 \\
\hline $\mathrm{C}(9)-\mathrm{C}(3)$ & 1.45 & $\mathrm{C}(6)-\mathrm{C}(5)-\mathrm{C}(4)$ & 120 & $\mathrm{C}(11)$ & 0.00 \\
\hline $\mathrm{C}(9)-\mathrm{C}(16)$ & 1.45 & $\mathrm{C}(13)-\mathrm{C}(14)-\mathrm{C}(5)$ & 120 & $\mathrm{C}(12)$ & -0.04 \\
\hline $\mathrm{C}(10)-\mathrm{C}(9)$ & 1.43 & $\mathrm{C}(34)-\mathrm{C}(14)-\mathrm{C}(5)$ & 119 & $\mathrm{C}(13)$ & -0.01 \\
\hline $\mathrm{C}(10)-\mathrm{C}(19)$ & 1.45 & $\mathrm{C}(14)-\mathrm{C}(5)-\mathrm{C}(6)$ & 120 & $\mathrm{C}(14)$ & -0.04 \\
\hline $\mathrm{C}(11)-\mathrm{C}(6)$ & 1.43 & $\mathrm{C}(1)-\mathrm{C}(2)-\mathrm{C}(7)$ & 122 & $\mathrm{C}(15)$ & -0.01 \\
\hline $\mathrm{C}(12)-\mathrm{C}(11)$ & 1.43 & $\mathrm{C}(10)-\mathrm{C}(8)-\mathrm{C}(7)$ & 121 & $\mathrm{C}(16)$ & -0.01 \\
\hline $\mathrm{C}(13)-\mathrm{C}(12)$ & 1.42 & $\mathrm{C}(2)-\mathrm{C}(7)-\mathrm{C}(8)$ & 122 & $\mathrm{C}(17)$ & -0.03 \\
\hline $\mathrm{C}(14)-\mathrm{C}(13)$ & 1.40 & $\mathrm{C}(9)-\mathrm{C}(10)-\mathrm{C}(8)$ & 118 & $\mathrm{C}(18)$ & -0.04 \\
\hline
\end{tabular}


Recent Advances in Petrochemical Science

\begin{tabular}{|c|c|c|c|c|c|}
\hline $\mathrm{C}(15)-\mathrm{C}(11)$ & 1.45 & $C(19)-C(10)-C(8)$ & 122 & $C(19)$ & -0.04 \\
\hline C(16)-C(15) & 1.44 & $C(2)-C(3)-C(9)$ & 120 & $\mathrm{C}(20)$ & -0.04 \\
\hline $\mathrm{C}(17)-\mathrm{C}(16)$ & 1.42 & $C(6)-C(3)-C(9)$ & 119 & $\mathrm{C}(21)$ & -0.04 \\
\hline$C(17)-C(24)$ & 1.43 & $C(15)-C(16)-C(9)$ & 120 & $\mathrm{C}(22)$ & -0.03 \\
\hline $\mathrm{C}(18)-\mathrm{C}(17)$ & 1.45 & $C(19)-C(10)-C(9)$ & 119 & $\mathrm{C}(23)$ & -0.04 \\
\hline C(19)-C(18) & 1.37 & $C(17)-C(16)-C(9)$ & 120 & $C(24)$ & -0.04 \\
\hline $\mathrm{C}(20)-\mathrm{C}(12)$ & 1.45 & $\mathrm{C}(3)-\mathrm{C}(9)-\mathrm{C}(10)$ & 120 & $\mathrm{H}(25)$ & +0.06 \\
\hline$C(21)-C(20)$ & 1.37 & $C(16)-C(9)-C(10)$ & 119 & $\mathrm{H}(26)$ & +0.06 \\
\hline$C(21)-C(22)$ & 1.45 & $\mathrm{C}(18)-\mathrm{C}(19)-\mathrm{C}(10)$ & 121 & $\mathrm{H}(27)$ & +0.06 \\
\hline$C(22)-C(15)$ & 1.42 & $C(5)-C(6)-C(11)$ & 120 & $\mathrm{H}(28)$ & +0.06 \\
\hline$C(23)-C(22)$ & 1.43 & $C(6)-C(11)-C(12)$ & 120 & $\mathrm{H}(29)$ & +0.06 \\
\hline$C(24)-C(23)$ & 1.38 & $\mathrm{C}(15)-\mathrm{C}(11)-\mathrm{C}(12)$ & 119 & $\mathrm{H}(30)$ & +0.06 \\
\hline $\mathrm{H}(25)-\mathrm{C}(7)$ & 1.09 & $C(11)-C(12)-C(13)$ & 119 & $\mathrm{H}(31)$ & +0.06 \\
\hline $\mathrm{H}(26)-\mathrm{C}(8)$ & 1.09 & $C(20)-C(12)-C(13)$ & 122 & $\mathrm{H}(32)$ & +0.06 \\
\hline $\mathrm{H}(27)-\mathrm{C}(19)$ & 1.09 & $C(12)-C(13)-C(14)$ & 122 & $\mathrm{H}(33)$ & +0.06 \\
\hline $\mathrm{H}(28)-\mathrm{C}(18)$ & 1.09 & $C(6)-C(11)-C(15)$ & 120 & $C(34)$ & -0.04 \\
\hline$H(29)-C(13)$ & 1.09 & $C(11)-C(15)-C(16)$ & 120 & C(35) & -0.04 \\
\hline $\mathrm{H}(30)-\mathrm{C}(20)$ & 1.09 & $C(22)-C(15)-C(16)$ & 120 & $C(36)$ & -0.04 \\
\hline $\mathrm{H}(31)-\mathrm{C}(21)$ & 1.09 & $C(24)-C(17)-C(16)$ & 119 & $\mathrm{C}(37)$ & -0.04 \\
\hline $\mathrm{H}(32)-\mathrm{C}(23)$ & 1.09 & $\mathrm{C}(15)-\mathrm{C}(16)-\mathrm{C}(17)$ & 120 & $\mathrm{C}(38)$ & -0.03 \\
\hline $\mathrm{H}(33)-\mathrm{C}(24)$ & 1.09 & $C(23)-C(24)-C(17)$ & 121 & C(39) & -0.06 \\
\hline $\mathrm{C}(34)-\mathrm{C}(14)$ & 1.46 & $\mathrm{C}(16)-\mathrm{C}(17)-\mathrm{C}(18)$ & 119 & $\mathrm{H}(40)$ & +0.06 \\
\hline$C(35)-C(34)$ & 1.36 & $\mathrm{C}(24)-\mathrm{C}(17)-\mathrm{C}(18)$ & 122 & $\mathrm{H}(41)$ & +0.06 \\
\hline$C(35)-C(36)$ & 1.46 & $\mathrm{C}(17)-\mathrm{C}(18)-\mathrm{C}(19)$ & 121 & $\mathrm{H}(42)$ & +0.06 \\
\hline $\mathrm{C}(36)-\mathrm{C}(4)$ & 1.44 & $\mathrm{C}(11)-\mathrm{C}(12)-\mathrm{C}(20)$ & 119 & $\mathrm{H}(43)$ & +0.06 \\
\hline $\mathrm{C}(37)-\mathrm{C}(39)$ & 1.40 & $\mathrm{C}(22)-\mathrm{C}(21)-\mathrm{C}(20)$ & 121 & $\mathrm{H}(44)$ & +0.06 \\
\hline $\mathrm{C}(37)-\mathrm{C}(1)$ & 1.42 & $C(12)-C(20)-C(21)$ & 121 & & \\
\hline$C(38)-C(36)$ & 1.41 & $C(15)-C(22)-C(21)$ & 119 & & \\
\hline $\mathrm{C}(39)-\mathrm{C}(38)$ & 1.40 & $C(23)-C(22)-C(21)$ & 122 & & \\
\hline $\mathrm{H}(40)-\mathrm{C}(34)$ & 1.09 & $\mathrm{C}(11)-\mathrm{C}(15)-\mathrm{C}(22)$ & 120 & & \\
\hline $\mathrm{H}(41)-\mathrm{C}(35)$ & 1.09 & $\mathrm{C}(15)-\mathrm{C}(22)-\mathrm{C}(23)$ & 119 & & \\
\hline $\mathrm{H}(42)-\mathrm{C}(38)$ & 1.09 & $\mathrm{C}(22)-\mathrm{C}(23)-\mathrm{C}(24)$ & 121 & & \\
\hline $\mathrm{H}(43)-\mathrm{C}(39)$ & 1.09 & $\mathrm{C}(2)-\mathrm{C}(7)-\mathrm{H}(25)$ & 120 & & \\
\hline \multirow[t]{13}{*}{$\mathrm{H}(44)-\mathrm{C}(37)$} & 1.09 & $\mathrm{C}(7)-\mathrm{C}(8)-\mathrm{H}(26)$ & 119 & & \\
\hline & & $\mathrm{C}(10)-\mathrm{C}(8)-\mathrm{H}(26)$ & 120 & & \\
\hline & & $\mathrm{C}(18)-\mathrm{C}(19)-\mathrm{H}(27)$ & 120 & & \\
\hline & & $\mathrm{C}(17)-\mathrm{C}(18)-\mathrm{H}(28)$ & 119 & & \\
\hline & & $\mathrm{C}(12)-\mathrm{C}(13)-\mathrm{H}(29)$ & 119 & & \\
\hline & & $\mathrm{C}(12)-\mathrm{C}(20)-\mathrm{H}(30)$ & 119 & & \\
\hline & & $\mathrm{C}(20)-\mathrm{C}(21)-\mathrm{H}(31)$ & 120 & & \\
\hline & & $\mathrm{C}(22)-\mathrm{C}(21)-\mathrm{H}(31)$ & 119 & & \\
\hline & & $\mathrm{C}(22)-\mathrm{C}(23)-\mathrm{H}(32)$ & 119 & & \\
\hline & & $\mathrm{C}(23)-\mathrm{C}(24)-\mathrm{H}(33)$ & 120 & & \\
\hline & & $\mathrm{C}(13)-\mathrm{C}(14)-\mathrm{C}(34)$ & 122 & & \\
\hline & & $C(36)-C(35)-C(34)$ & 122 & & \\
\hline & & $C(14)-C(34)-C(35)$ & 121 & & \\
\hline
\end{tabular}


Recent Advances in Petrochemical Science

\begin{tabular}{|l|c|c|c|l|l|}
\hline & & $\mathrm{C}(4)-\mathrm{C}(36)-\mathrm{C}(35)$ & 119 & & \\
\hline & $\mathrm{C}(38)-\mathrm{C}(36)-\mathrm{C}(35)$ & 121 & & \\
\hline & $\mathrm{C}(1)-\mathrm{C}(4)-\mathrm{C}(36)$ & 120 & & \\
\hline & $\mathrm{C}(5)-\mathrm{C}(4)-\mathrm{C}(36)$ & 119 & & \\
\hline & $\mathrm{C}(38)-\mathrm{C}(39)-\mathrm{C}(37)$ & 120 & & \\
\hline & $\mathrm{C}(2)-\mathrm{C}(1)-\mathrm{C}(37)$ & 123 & & \\
\hline & $\mathrm{C}(4)-\mathrm{C}(36)-\mathrm{C}(38)$ & 120 & & \\
\hline & $\mathrm{C}(36)-\mathrm{C}(38)-\mathrm{C}(39)$ & 120 & & \\
\hline & $\mathrm{C}(14)-\mathrm{C}(34)-\mathrm{H}(40)$ & 118 & & \\
\hline & $\mathrm{C}(34)-\mathrm{C}(35)-\mathrm{H}(41)$ & 120 & & \\
\hline & $\mathrm{C}(36)-\mathrm{C}(35)-\mathrm{H}(41)$ & 118 & & \\
\hline & $\mathrm{C}(36)-\mathrm{C}(38)-\mathrm{H}(42)$ & 121 & & \\
\hline & & $\mathrm{C}(38)-\mathrm{C}(39)-\mathrm{H}(43)$ & 120 & & \\
\hline & & $\mathrm{C}(39)-\mathrm{C}(37)-\mathrm{H}(44)$ & 117 & & \\
\hline & & $\mathrm{C}(1)-\mathrm{C}(37)-\mathrm{H}(44)$ & 121 & & \\
\hline
\end{tabular}

Table 3: Optimized bond lengths, valence angles and atom charges of the the non-linear graphene of 10 hexagons.

\begin{tabular}{|c|c|c|c|c|c|}
\hline Bond Lengths & $\mathbf{R}, \mathbf{A}$ & Valence Angles & Grad & Atom & Atomic Charge \\
\hline $\mathrm{C}(2)-\mathrm{C}(1)$ & 1.46 & $\mathrm{C}(5)-\mathrm{C}(4)-\mathrm{C}(1)$ & 120 & $\mathrm{C}(1)$ & -0.01 \\
\hline $\mathrm{C}(3)-\mathrm{C}(2)$ & 1.43 & $\mathrm{C}(38)-\mathrm{C}(36)-\mathrm{C}(1)$ & 119 & $\mathrm{C}(2)$ & 0.00 \\
\hline $\mathrm{C}(3)-\mathrm{C}(6)$ & 1.46 & $\mathrm{C}(44)-\mathrm{C}(36)-\mathrm{C}(1)$ & 119 & $\mathrm{C}(3)$ & -0.01 \\
\hline $\mathrm{C}(4)-\mathrm{C}(1)$ & 1.44 & $\mathrm{C}(6)-\mathrm{C}(3)-\mathrm{C}(2)$ & 120 & $\mathrm{C}(4)$ & -0.01 \\
\hline $\mathrm{C}(4)-\mathrm{C}(5)$ & 1.46 & $\mathrm{C}(1)-\mathrm{C}(2)-\mathrm{C}(3)$ & 120 & $\mathrm{C}(5)$ & 0.00 \\
\hline $\mathrm{C}(5)-\mathrm{C}(14)$ & 1.44 & $\mathrm{C}(5)-\mathrm{C}(6)-\mathrm{C}(3)$ & 120 & $\mathrm{C}(6)$ & -0.01 \\
\hline $\mathrm{C}(6)-\mathrm{C}(5)$ & 1.43 & $\mathrm{C}(7)-\mathrm{C}(2)-\mathrm{C}(3)$ & 120 & $\mathrm{C}(7)$ & -0.04 \\
\hline $\mathrm{C}(7)-\mathrm{C}(2)$ & 1.44 & $\mathrm{C}(11)-\mathrm{C}(6)-\mathrm{C}(3)$ & 120 & $\mathrm{C}(8)$ & -0.01 \\
\hline $\mathrm{C}(8)-\mathrm{C}(7)$ & 1.41 & $\mathrm{C}(10)-\mathrm{C}(9)-\mathrm{C}(3)$ & 120 & $\mathrm{C}(9)$ & 0.00 \\
\hline $\mathrm{C}(9)-\mathrm{C}(3)$ & 1.43 & $\mathrm{C}(16)-\mathrm{C}(9)-\mathrm{C}(3)$ & 120 & $\mathrm{C}(10)$ & -0.04 \\
\hline $\mathrm{C}(9)-\mathrm{C}(10)$ & 1.44 & $\mathrm{C}(2)-\mathrm{C}(1)-\mathrm{C}(4)$ & 120 & $\mathrm{C}(11)$ & 0.00 \\
\hline $\mathrm{C}(9)-\mathrm{C}(16)$ & 1.46 & $\mathrm{C}(14)-\mathrm{C}(5)-\mathrm{C}(4)$ & 120 & $\mathrm{C}(12)$ & -0.04 \\
\hline $\mathrm{C}(10)-\mathrm{C}(8)$ & 1.41 & $\mathrm{C}(6)-\mathrm{C}(5)-\mathrm{C}(4)$ & 120 & $\mathrm{C}(13)$ & -0.01 \\
\hline $\mathrm{C}(10)-\mathrm{C}(19)$ & 1.45 & $\mathrm{C}(13)-\mathrm{C}(14)-\mathrm{C}(5)$ & 119 & $\mathrm{C}(14)$ & -0.04 \\
\hline $\mathrm{C}(11)-\mathrm{C}(6)$ & 1.43 & $\mathrm{C}(33)-\mathrm{C}(14)-\mathrm{C}(5)$ & 118 & $\mathrm{C}(15)$ & -0.01 \\
\hline $\mathrm{C}(12)-\mathrm{C}(11)$ & 1.44 & $\mathrm{C}(14)-\mathrm{C}(5)-\mathrm{C}(6)$ & 120 & $\mathrm{C}(16)$ & -0.01 \\
\hline $\mathrm{C}(13)-\mathrm{C}(12)$ & 1.41 & $\mathrm{C}(1)-\mathrm{C}(2)-\mathrm{C}(7)$ & 120 & $\mathrm{C}(17)$ & -0.03 \\
\hline $\mathrm{C}(14)-\mathrm{C}(13)$ & 1.41 & $\mathrm{C}(2)-\mathrm{C}(7)-\mathrm{C}(8)$ & 119 & $\mathrm{C}(18)$ & -0.04 \\
\hline $\mathrm{C}(15)-\mathrm{C}(11)$ & 1.46 & $\mathrm{C}(43)-\mathrm{C}(7)-\mathrm{C}(8)$ & 123 & $\mathrm{C}(19)$ & -0.04 \\
\hline $\mathrm{C}(16)-\mathrm{C}(15)$ & 1.44 & $\mathrm{C}(19)-\mathrm{C}(10)-\mathrm{C}(8)$ & 123 & $\mathrm{C}(20)$ & -0.04 \\
\hline $\mathrm{C}(17)-\mathrm{C}(16)$ & 1.43 & $\mathrm{C}(2)-\mathrm{C}(3)-\mathrm{C}(9)$ & 120 & $\mathrm{C}(21)$ & -0.04 \\
\hline $\mathrm{C}(17)-\mathrm{C}(24)$ & 1.43 & $\mathrm{C}(6)-\mathrm{C}(3)-\mathrm{C}(9)$ & 120 & $\mathrm{C}(22)$ & -0.03 \\
\hline $\mathrm{C}(18)-\mathrm{C}(17)$ & 1.45 & $\mathrm{C}(8)-\mathrm{C}(10)-\mathrm{C}(9)$ & 119 & $\mathrm{C}(23)$ & -0.04 \\
\hline $\mathrm{C}(19)-\mathrm{C}(18)$ & 1.37 & $\mathrm{C}(19)-\mathrm{C}(10)-\mathrm{C}(9)$ & 118 & $\mathrm{C}(24)$ & -0.04 \\
\hline $\mathrm{C}(20)-\mathrm{C}(12)$ & 1.45 & $\mathrm{C}(15)-\mathrm{C}(16)-\mathrm{C}(9)$ & 120 & $\mathrm{H}(25)$ & +0.06 \\
\hline $\mathrm{C}(21)-\mathrm{C}(20)$ & 1.37 & $\mathrm{C}(17)-\mathrm{C}(16)-\mathrm{C}(9)$ & 120 & $\mathrm{H}(26)$ & +0.06 \\
\hline $\mathrm{C}(21)-\mathrm{C}(22)$ & 1.45 & $\mathrm{C}(7)-\mathrm{C}(8)-\mathrm{C}(10)$ & 122 & $\mathrm{H}(27)$ & +0.06 \\
\hline $\mathrm{C}(22)-\mathrm{C}(15)$ & 1.43 & $\mathrm{C}(18)-\mathrm{C}(19)-\mathrm{C}(10)$ & 122 & $\mathrm{H}(28)$ & +0.06 \\
\hline
\end{tabular}


Recent Advances in Petrochemical Science

\begin{tabular}{|c|c|c|c|c|c|}
\hline$C(23)-C(22)$ & 1.43 & $C(16)-C(9)-C(10)$ & 120 & $\mathrm{H}(29)$ & +0.06 \\
\hline$C(24)-C(23)$ & 1.39 & $C(5)-C(6)-C(11)$ & 120 & $\mathrm{H}(30)$ & +0.06 \\
\hline $\mathrm{H}(25)-\mathrm{C}(8)$ & 1.09 & $C(6)-C(11)-C(12)$ & 120 & $\mathrm{H}(31)$ & +0.06 \\
\hline$H(26)-C(19)$ & 1.09 & $C(15)-C(11)-C(12)$ & 120 & $\mathrm{H}(32)$ & +0.06 \\
\hline $\mathrm{H}(27)-\mathrm{C}(18)$ & 1.09 & $\mathrm{C}(11)-\mathrm{C}(12)-\mathrm{C}(13)$ & 119 & C(33) & -0.04 \\
\hline $\mathrm{H}(28)-\mathrm{C}(13)$ & 1.09 & $C(20)-C(12)-C(13)$ & 123 & C(34) & -0.04 \\
\hline $\mathrm{H}(29)-\mathrm{C}(20)$ & 1.09 & $C(12)-C(13)-C(14)$ & 122 & C(35) & -0.03 \\
\hline $\mathrm{H}(30)-\mathrm{C}(21)$ & 1.09 & $C(6)-C(11)-C(15)$ & 120 & $\mathrm{C}(36)$ & -0.03 \\
\hline $\mathrm{H}(31)-\mathrm{C}(23)$ & 1.09 & $C(11)-C(15)-C(16)$ & 120 & C(37) & -0.04 \\
\hline $\mathrm{H}(32)-\mathrm{C}(24)$ & 1.09 & $\mathrm{C}(22)-\mathrm{C}(15)-\mathrm{C}(16)$ & 120 & C(38) & -0.04 \\
\hline$C(33)-C(14)$ & 1.45 & $C(24)-C(17)-C(16)$ & 119 & $\mathrm{H}(39)$ & +0.06 \\
\hline$C(34)-C(33)$ & 1.37 & $C(15)-C(16)-C(17)$ & 120 & $\mathrm{H}(40)$ & +0.06 \\
\hline$C(34)-C(35)$ & 1.45 & $C(23)-C(24)-C(17)$ & 121 & $\mathrm{H}(41)$ & +0.06 \\
\hline$C(35)-C(4)$ & 1.43 & $C(16)-C(17)-C(18)$ & 119 & $\mathrm{H}(42)$ & +0.06 \\
\hline$C(36)-C(1)$ & 1.43 & $\mathrm{C}(24)-\mathrm{C}(17)-\mathrm{C}(18)$ & 123 & $\mathrm{C}(43)$ & -0.04 \\
\hline$C(36)-C(38)$ & 1.43 & $C(17)-C(18)-C(19)$ & 122 & $\mathrm{C}(44)$ & -0.04 \\
\hline$C(36)-C(44)$ & 1.45 & $C(11)-C(12)-C(20)$ & 118 & $\mathrm{H}(45)$ & +0.06 \\
\hline$C(37)-C(35)$ & 1.43 & $C(22)-C(21)-C(20)$ & 121 & $\mathrm{H}(46)$ & +0.06 \\
\hline $\mathrm{C}(38)-\mathrm{C}(37)$ & 1.39 & $C(12)-C(20)-C(21)$ & 122 & & \\
\hline $\mathrm{H}(39)-\mathrm{C}(33)$ & 1.09 & $C(15)-C(22)-C(21)$ & 119 & & \\
\hline $\mathrm{H}(40)-\mathrm{C}(34)$ & 1.09 & $\mathrm{C}(23)-\mathrm{C}(22)-\mathrm{C}(21)$ & 123 & & \\
\hline $\mathrm{H}(41)-\mathrm{C}(37)$ & 1.09 & $\mathrm{C}(11)-\mathrm{C}(15)-\mathrm{C}(22)$ & 120 & & \\
\hline $\mathrm{H}(42)-\mathrm{C}(38)$ & 1.09 & $C(15)-C(22)-C(23)$ & 119 & & \\
\hline $\mathrm{C}(43)-\mathrm{C}(7)$ & 1.45 & $C(22)-C(23)-C(24)$ & 121 & & \\
\hline$C(44)-C(43)$ & 1.37 & $\mathrm{C}(7)-\mathrm{C}(8)-\mathrm{H}(25)$ & 119 & & \\
\hline $\mathrm{H}(45)-\mathrm{C}(44)$ & 1.09 & $\mathrm{C}(18)-\mathrm{C}(19)-\mathrm{H}(26)$ & 120 & & \\
\hline \multirow[t]{20}{*}{$\mathrm{H}(46)-\mathrm{C}(43)$} & 1.09 & $\mathrm{C}(17)-\mathrm{C}(18)-\mathrm{H}(27)$ & 118 & & \\
\hline & & $\mathrm{C}(12)-\mathrm{C}(13)-\mathrm{H}(28)$ & 119 & & \\
\hline & & $\mathrm{C}(12)-\mathrm{C}(20)-\mathrm{H}(29)$ & 118 & & \\
\hline & & $\mathrm{C}(20)-\mathrm{C}(21)-\mathrm{H}(30)$ & 120 & & \\
\hline & & $\mathrm{C}(22)-\mathrm{C}(21)-\mathrm{H}(30)$ & 118 & & \\
\hline & & $\mathrm{C}(22)-\mathrm{C}(23)-\mathrm{H}(31)$ & 120 & & \\
\hline & & $\mathrm{C}(23)-\mathrm{C}(24)-\mathrm{H}(32)$ & 119 & & \\
\hline & & $C(13)-C(14)-C(33)$ & 123 & & \\
\hline & & $C(35)-C(34)-C(33)$ & 121 & & \\
\hline & & $C(14)-C(33)-C(34)$ & 122 & & \\
\hline & & $C(4)-C(35)-C(34)$ & 119 & & \\
\hline & & $C(37)-C(35)-C(34)$ & 123 & & \\
\hline & & $C(1)-C(4)-C(35)$ & 120 & & \\
\hline & & $C(5)-C(4)-C(35)$ & 120 & & \\
\hline & & $C(2)-C(1)-C(36)$ & 120 & & \\
\hline & & $\mathrm{C}(37)-\mathrm{C}(38)-\mathrm{C}(36)$ & 121 & & \\
\hline & & $C(43)-C(44)-C(36)$ & 122 & & \\
\hline & & $C(4)-C(35)-C(37)$ & 119 & & \\
\hline & & $\mathrm{C}(35)-\mathrm{C}(37)-\mathrm{C}(38)$ & 121 & & \\
\hline & & $C(44)-C(36)-C(38)$ & 123 & & \\
\hline
\end{tabular}




\section{Recent Advances in Petrochemical Science}

\begin{tabular}{|l|c|c|c|c|c|}
\hline & & $\mathrm{C}(14)-\mathrm{C}(33)-\mathrm{H}(39)$ & 118 & & \\
\hline & & $\mathrm{C}(33)-\mathrm{C}(34)-\mathrm{H}(40)$ & 120 & & \\
\hline & & $\mathrm{C}(35)-\mathrm{C}(34)-\mathrm{H}(40)$ & 118 & & \\
\hline & & $\mathrm{C}(35)-\mathrm{C}(37)-\mathrm{H}(41)$ & 119 & & \\
\hline & & $\mathrm{C}(37)-\mathrm{C}(38)-\mathrm{H}(42)$ & 119 & & \\
\hline & & $\mathrm{C}(2)-\mathrm{C}(7)-\mathrm{C}(43)$ & 118 & & \\
\hline & & $\mathrm{C}(7)-\mathrm{C}(43)-\mathrm{C}(44)$ & 122 & & \\
\hline & & $\mathrm{C}(43)-\mathrm{C}(44)-\mathrm{H}(45)$ & 120 & & \\
\hline
\end{tabular}

Table 4: Total energy $\left(\mathrm{E}_{0}\right)$, maximal charge on atoms (qmax $\left.{ }^{\mathrm{H}}\right)$, acid strength $(\mathrm{pKa})$, the highest occupied molecular orbital energy $\left(\mathrm{E}_{\mathrm{HOMO}}\right)$, the lowest unoccupied molecular orbital energy $\left(E_{L_{u m o}}\right)$, the Hartree energy $\left(E_{H}\right)$ of the graphenes consisting of 7,9 and 10 hexagons.

\begin{tabular}{|c|c|c|c|c|c|c|c|}
\hline Graphene & $\mathbf{E}_{\mathbf{0}}(\mathbf{K j} / \mathbf{M o l})$ & $\mathbf{Q m a x}^{\mathbf{h +}}$ & $\mathbf{P k a}$ & $\mathbf{E}_{\text {LUMo }}(\mathbf{K j} / \mathbf{M o l})$ & $\mathbf{E}_{\text {LUMo }}(\mathbf{K j} / \mathbf{M o l})$ & $\mathbf{E}_{\mathrm{H}}(\mathbf{K j} / \mathbf{M o l})$ & $\mathbf{E}_{\mathrm{H}}(\mathbf{E v})$ \\
\hline 7 hexagons & -312188 & 0,06 & 33 & -98 & -779 & 681 & 7,1 \\
\hline 9 hexagons & -388846 & 0,06 & 33 & -130 & -744 & 614 & 6,4 \\
\hline 10 hexagons & -413507 & 0,06 & 33 & -146 & -729 & 583 & 6 \\
\hline
\end{tabular}

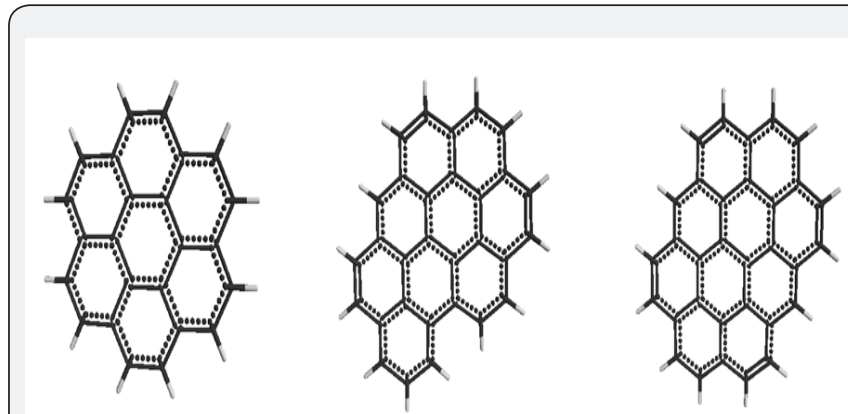

Figure 1: The schemes of the graphenes of 7,9 and 10 hexagons.

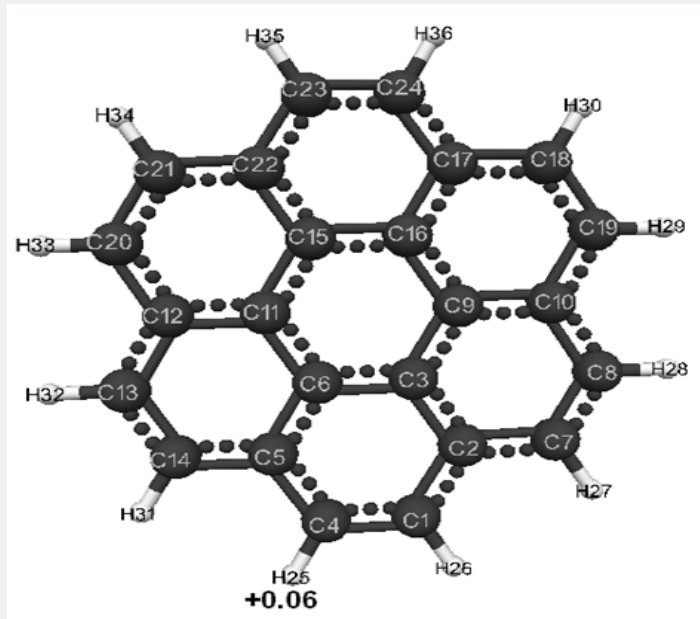

Figure 2: Geometric and electronic structure of the non-linear graphene of 7 hexagons.

$\left(E_{0}=-312186 \mathrm{~kJ} / \mathrm{mol}, E_{E L}=-2373711 \mathrm{~kJ} / \mathrm{mol}\right)$

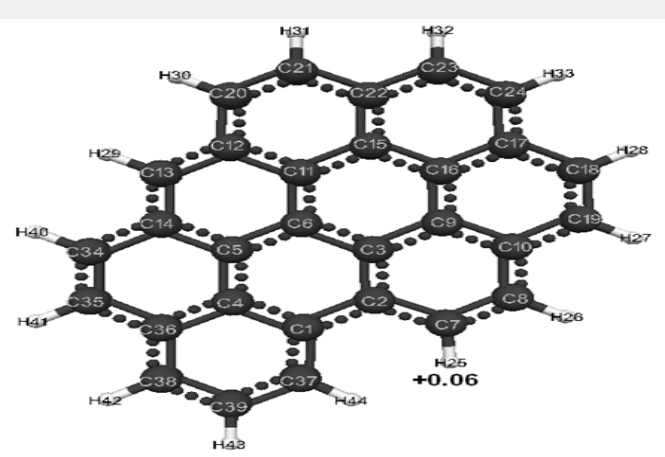

Figure 3: Geometric and electronic structure of the non-linear graphene of 9 hexagons.

$\left(E_{0}=-388844 \mathrm{~kJ} / \mathrm{mol}, E_{E L}=-3301699 \mathrm{~kJ} / \mathrm{mol}\right)$

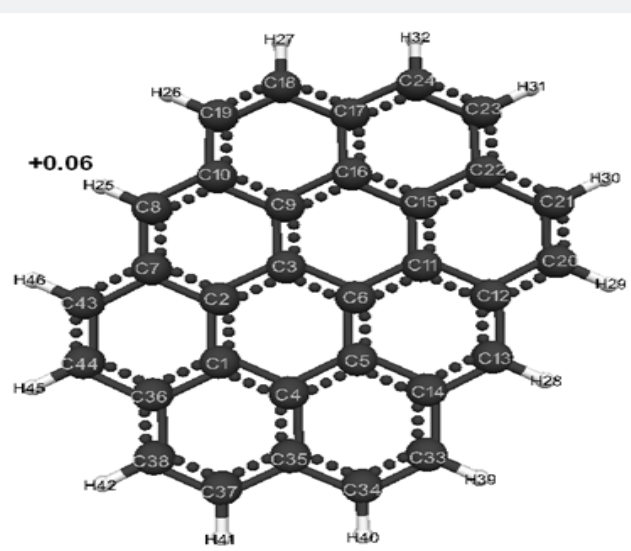

Figure 4: Geometric and electronic structure of the non-linear graphene of 10 hexagons.

$\left(E_{0}=-413506 \mathrm{~kJ} / \mathrm{mol}, E_{E L}=-3632286 \mathrm{~kJ} / \mathrm{mol}\right)$ 


\section{Conclusion}

Thus, we performed the quantum-chemical calculation of molecules of non-linear graphenes consisting of 7, 9 and 10 hexagons by the MNDO method for the first time. Their optimized geometric and electronic structure is obtained. The acid strength of these graphenes is theoretically estimated: $\mathrm{pKa}=33$. It is established that the molecules of 7, 9 and 10 hexagons belong to the class of very weak acids ( $\mathrm{pKa}>14$ ). An increase in the number of hexagons in nonlinear graphenes reduces the Hartree energy, which coincides with the trend noted in [1].

\section{References}

1. Babkin VA, Andreev DS, Tarasov EA, Fedunov RG, Lebedev NG et al. (2017) Quantum-chemical modeling of 1,12-benzperylene, 2,3-benzoperylene and perylene in the framework of the molecular nonlinear graphene model by the MNDO method. Izvestiya of the Volgograd State Technical University. Series "Chemistry and Technology of Organ element Monomers and Polymer Materials 4(199): 21-28.

2. Tsirelson VG (2010) Quantum Chemistry Molecules molecular systems and solids. ( $3^{\text {rd }}$ edn), Binom, Moscow, Russia, p. 496.

3. http://classic.chem.msu.su/gran/firefly/index.html

4. Schmidt MW, Baldrosge KK, Elbert JA, Gordon MS (1993) General Atomic and Molecular Electronic Structure Systems. J Comput Chem 14: $1347-1363$

5. Babkin VA, Fedunov RG, Minsker KS, Ponomarev OA, Yu A, et al. (2002) Connection of the universal acidity index of $\mathrm{H}$-acids with the charge on hydrogen atom (AB INITIO method). Oxidation Communications 25(1): 21-47.

6. Bode BM, Gordon MS (1998) Mac MolPlt: A Graphical User Interface for GAMESS. J Molec Graphics 16(3): 133-138.

7. Clar E (1971) Poly cyclic hydrocarbons. Chemistry, E Clar, Moscow, Russia, p. 442.

\section{Your next submission with Juniper Publishers will reach you the below assets}

- Quality Editorial service

- Swift Peer Review

- Reprints availability

- E-prints Service

- Manuscript Podcast for convenient understanding

- Global attainment for your research

- Manuscript accessibility in different formats

( Pdf, E-pub, Full Text, Audio)

- Unceasing customer service

Track the below URL for one-step submission https://juniperpublishers.com/online-submission.php 